\title{
Current diagnostic approach and screening methods for hereditary spherocytosis
}

\author{
Paola Bianchi \\ Hematology and Transplant Unit, Pathophysiology of Anaemia Unit, Foundation IRCCS Ca'
Granda Ospedale Maggiore Policlinico, Milan, Italy
}

The plasma membrane of the erythrocyte accounts for all of its antigenic, transport, and mechanical characteristics, particularly its ability to undergo large passive deformations during repeated passage through the narrow capillaries of the microvasculature, throughout its 120-day life span. The determinant of normal membrane cohesion is the system of vertical linkages between phospholipid bilayer and membrane skeleton, formed by the interactions of the cytoplasmic domains of various membrane proteins with the spectrin-based skeletal network. Band 3 and RhAG provide such links by interacting with ankyrin, which in turn binds to $\beta$-spectrin. Protein 4.2 binds to both band 3 and ankyrin and can regulate the avidity of the interaction between band 3 and ankyrin. Glycophorin C, band 3, XK, Rh, and Duffy all bind to protein $4.1 \mathrm{R}$, the third member of the ternary junctional complex with $\beta$ spectrin and actin. Horizontal linkages in the membrane skeleton due to spectrin dimer-dimer interaction or spectrin-actin-protein 4.1 junctional complex, confer to the erythrocytes elasticity and deformability (Figure 1) (1-2).

Hereditary spherocytosis (HS) is the most common congenital red cell membrane defect that occurs in all racial groups and is particularly common in individuals of northern European ancestry. Its prevalence, considered in the past to be 1:5000, is more properly estimated to be 1:2000 based on studies of decreased erythrocyte osmotic fragility in blood donors (3).

Approximately $75 \%$ of cases display an autosomal dominant pattern of inheritance, the remaining comprising recessive forms and de novo mutations (4). The main clinical features of HS are haemolytic anaemia from compensated to severe, sometimes requiring exchange transfusion at birth and/or repeated blood transfusions, variable jaundice, splenomegaly and cholelythiasis (2). The molecular defect is highly heterogeneous, involving the genes encoding for spectrin, ankyrin, band 3 and protein 4.2 . The deficiency or dysfunction of any

Correspondence: Paola Bianchi

E-mail: paola.bianchi@policlinico.mi.it

(C) Copyright P. Bianchi, 2013

Licensee PAGEPress, Italy

Thalassemia Reports 2013; 3(s1):e32

doi:10.4081/thal.2013.s1.e32

This article is distributed under the terms of the Creative Commons Attribution Noncommercial License (by-nc 3.0) which permits any noncommercial use, distribution, and reproduction in any medium, provided the original author(s) and source are credited.

Parts of this work were presented at the

"3rd Pan-European Conference on Haemoglobinopathies and Rare Anaemias", Limassol (Cyprus), 24-26 October 2012. of these proteins, which are involved in the attachment of cytoskeleton to the membrane integral domain, results in a loss of surface area and leads to spheroidal, osmotically fragile cells that are selectively trapped in the spleen.

The defective protein can be detected by sodium dodecyl sulphatepolyacrylamide gel electrophoresis (SDS-PAGE) that allows the identification of different subsets of patients; a portion of HS subjects remains however unclassified by this technique.

The analysis of a large database of 300 Italian HS patients (5), showed that band 3 and spectrin deficiency were the most common protein abnormalities ( $54 \%$ and $31 \%$, respectively). Spectrin deficiency was more frequently diagnosed in childhood and band 3 in adulthood. Splenomegaly and gallstones were more frequent in band 3 deficient patients, whereas anaemia, neonatal jaundice and transfusion requirement were more common in spectrin/ankyrin deficiency. Consistently, haemoglobin was slightly lower, spherocytes number and haemolysis markers higher in spectrin than in band 3 deficiency. Splenectomy corrected the anaemia in all cases but one with severe spectrin deficiency; in addition, after splenectomy spectrin deficient patients showed a slightly lower median rise in haemoglobin, and a higher reticulocyte number than band 3 deficient once, suggesting a more severe clinical pattern in the former.

The diagnosis of HS is the final step of a diagnostic workout based not only on laboratory tests but also on clinical examination, personal family history, and the exclusion of possible causes of secondary spherocytosis. However, given the rarity and the wide clinical heterogeneity, the diagnosis of these defects can be difficult, in particular in mild and atypical forms.

The typical laboratory hallmark of HS, although not specific, is the presence of spherocytes on peripheral blood smear (6). By the analysis of a large series of HS patients (5) median spherocytes number was $7 \%$ (range $0-56$ ); $8 \%$ of the patients had less than $2 \%$ spherocytes and $3 \%$ had no detectable spherocytes. Spherocytes number in fact, may be very few in several patients requiring skilled operators to be detected; moreover, the microscopic examination of red cells is increasingly omitted in an era of laboratory automation.

The laboratory diagnosis of HS therefore commonly relies on tests that exploit the surface area-to-volume ratio, typically reduced in spherical-shaped erythrocytes, in particular the red cell osmotic fragility (OF) tests at various sodium chloride $(\mathrm{NaCl})$ concentrations on fresh and incubated blood, and assays that measure the extent or the rate of lysis of red cells suspended in buffered glycerol solutions, i.e. Glycerol Lysis (GLT), Acidified Glycerol Lysis (AGLT) and Pink test. However, these tests miss a variable portion of HS cases, particularly the mildest ones, and do not differentiate HS from secondary spherocytosis associated with other conditions, mainly autoimmune haemolytic anaemias. The cryohemolysis test, based on the observation that $\mathrm{HS}$ red cells are particularly susceptible to cooling at $0^{\circ} \mathrm{C}$ in hypertonic conditions, and the flow cytometric analysis of eosin-5'maleimide-labelled intact red blood cells (EMA-binding test) (7) have 
been proposed as additional method of identifying HS (8). The latter in particular, has been proven to be a sensitive and specific diagnostic tool for HS directly targeting the structural lesion of this disease, since the fluorescent probe eosin-5'-maleimide interacts with the protein band 3 complex $(7,9,10)$.

A recent study (11) has compared the performances of EMA-binding, NaCl-osmotic fragility on fresh and incubated blood, GLT, AGLT, and Pink test on a series of 150 patients with hereditary spherocytosis grouped according to the clinical phenotype and the defective protein, with the final aim to find the combination of tests associated with the highest diagnostic power, even in the mildest cases. It was confirmed that each individual test fails to diagnose a portion of cases. In particular, EMA-binding displayed $93 \%$ sensitivity and $98 \%$ specificity: sensitivity was independent of the type and amount of molecular defect and of clinical phenotype. A comparable sensitivity was shown by AGLT (95\%) and Pink test (91\%). The sensitivity of $\mathrm{NaCl}$ osmotic fragility tests, commonly considered the gold standard for diagnosis of hereditary spherocytosis, was $68 \%$ on fresh and $81 \%$ on incubated blood, and further decreased in compensated cases (53\% and 64\%). The association of EMA-binding and AGLT allowed to identify all the hereditary spherocytosis patients of the examined series and therefore may represent at present an effective diagnostic tool for HS also in mild/compensated cases.

In atypical HS cases, the diagnostic workout may be more complex requiring specific diagnostic tools such as SDS-PAGE of red cell membrane proteins or ekacytometry. SDS-PAGE analysis is also required when a congenital dyserythropoietic anaemia type II is suspected. It is worth mentioning that none of the available diagnostic tests for HS, neither direct or indirect, differentiates HS from CDAII, this latter, although less prevalent than HS, may mimic HS both in terms of clinical presentation and increased red cell osmotic fragility, and can be distinguished by HS through the identification of hypoglycosylation of band 3 . It was observed that $13 \%$ of cases referred to a reference laboratory with the provisional label of HS were found to be CDAII when examined by SDS-PAGE (5). Finally, the use of molecular testing in diagnosis of hereditary spherocytosis is at present indicated only in a few selected cases.

During the course of the ENERCA (European NEtwork for Rare Congenital Anemias) project specific surveys were promoted aimed to assess in a realistic way the adequacy of the different European hematologic services involved in the diagnosis, clinical care and external quality assessment for Rare anaemias. Among them a specific questionnaire on laboratory tests was circulated aimed at understanding which diagnostic tests/parameters were used for the diagnosis of red cell membrane disorders with the final aim to define a consensus among laboratories. Twenty-five European centres answered to the questionnaire. Although the results should be taken with caution since a limited number of Centres was involved and errors in the interpretation of the answers is always possible, some information can be retrieved. Beside red cell morphology and reticulocytes, osmotic fragility tests performed singularly or in combination are used in about $50 \%$ of the centres. Cryohemolysis test, albeit suggested as one of as reference test in guidelines for HS published by Bolton-Maggs (2004) is indeed used only in $20 \%$ of the Centres. Flow cytometric EMA-binding test seems to be the most commonly used test, performed in $60 \%$ of centres in all cases with suspected hemolytic anemia. Finally, SDSPAGE analysis, ektacytometry, and molecular analysis are performed only in selected atypical cases (Figure 2).

It is worth of noting that there is a wide heterogeneity of opinions about the methods with the best specificity and sensitivity to use in the diagnosis of red cell membrane disorders (Figure 3); in any case different combinations of tests, greatly variable from Centre to Centre, are used to reach a final diagnosis.
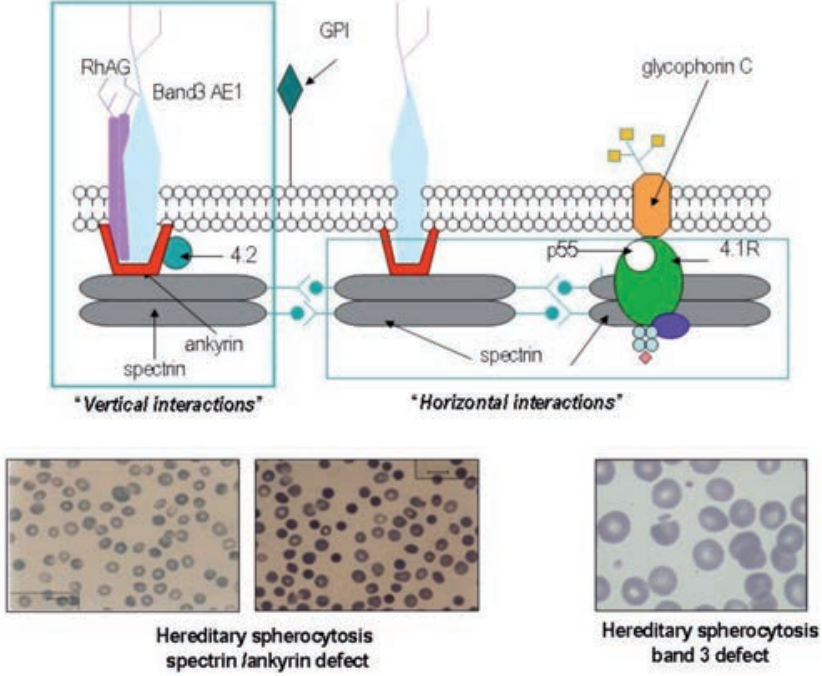

Figure 1. Schematical representation of RBC membrane structure, vertical and horizontal interactions. $\mathrm{RBC}$ morphology in hereditary spherocytosis.

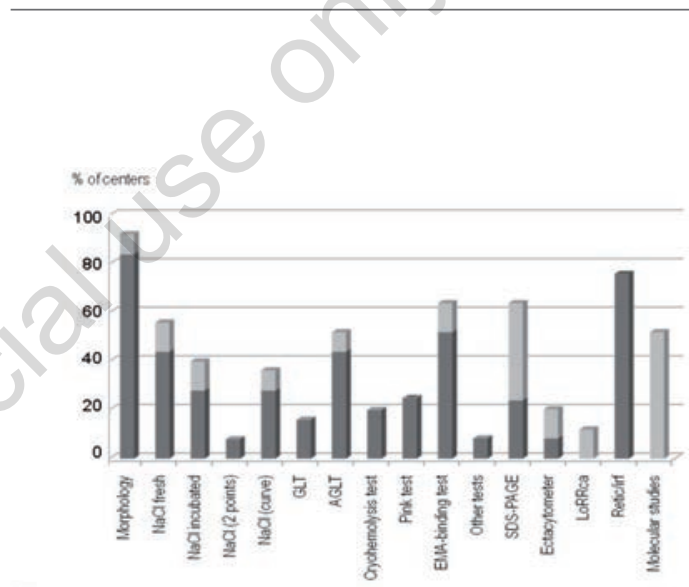

1. Perfomedinpatcularcoses

Aways petormed

enerca

Figure 2. Use of diagnostic tests performed for diagnosis of red cell membrane defects.

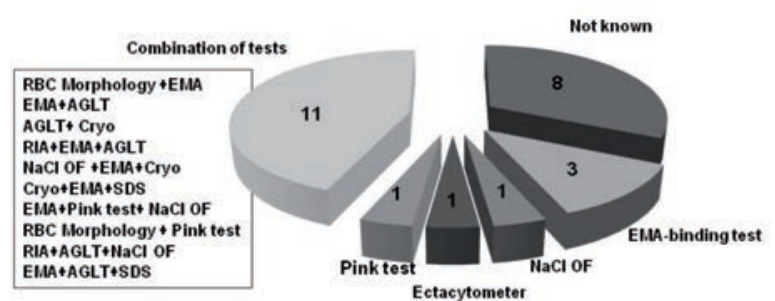

enerca

Figure 3. Method considered to have the best specificity and sensitivity, as resulted from the ENERCA survey on Diagnosis of RBC membrane disorders, 25 Centres involved. 


\section{References}

1. Mohandas N, and Gallagher PG. Red cell membrane: past, present, and future. Blood 2008; 112: 3939-48.

2. Perrotta S, Gallagher PG, Mohandas N. Hereditary spherocytosis. Lancet 2008; 372: 1411-26.

3. Eber SW, Pekrun A, Neufeldt A, Schröter W. Prevalence of increased osmotic fragility of erythrocytes in German blood donors: screening using a modified glycerol lysis test. Ann Hematol. 1992; 64(2):8892.

4. Delaunay J. The molecular basis of hereditary red cell membrane disorders. Blood Rev. 2007; 21(1):1-20.

5. Mariani M, Barcellini W, Vercellati C et al. Clinical and hematologic features of 300 patients affected by hereditary spherocytosis grouped according to the type of the membrane protein defect. Haematologica 2008; 93: 1310-7.

6. Grace RF, Lux SE. Disorders of red cell membrane. In: Orkin SH, Nathan DG, Ginsburg D, Fisher DE, Lux SE (eds). Hematology of infancy ad childhood. Saunders, Philadelphia, 2009 pp 659-838..

7. King MJ, Behrens J, Rogers C et al. Rapid flow cytometric test for the diagnosis of membrane cytoskeleton-associated haemolytic anaemia. Br J Haematol 2000; 111: 924-33.

8. Bolton-Maggs PH, Langer JC, Iolascon A, Tittensor P, King MJ.Guidelines for the diagnosis and management of hereditary spherocytosis - 2011 update. Br J Haematol. 2011; 156, 37-49.

9. Girodon F, Garçon L, Bergoin E, Largier M, Delaunay J, FénéantThibault M, et al. Usefulness of the eosin-5'-maleimide cytometric method as a first-line screening test for the diagnosis of hereditary spherocytosis: comparison with ektacytometry and protein electrophoresis. Br J Haematol. 2008;140(4):468-70.

10. Crisp RL, Solari L, Vota D, García E, Miguez G, Chamorro ME, et al. A prospective study to assess the predictive value for hereditary spherocytosis using five laboratory tests (cryohemolysis test, eosin-5'-maleimide flow cytometry, osmotic fragility test, autohemolysis test, and SDS-PAGE) on 50 hereditary spherocytosis families in Argentina. Ann Hematol. 2011; 90: 625-34.

11. Bianchi P, Fermo E, Vercellati C, Marcello AP, Porretti L, Cortelezzi A, Barcellini W, Zanella A Diagnostic power of laboratory tests for hereditary spherocytosis: a comparison study on 150 patients grouped according to the molecular and clinical characteristics. Haematologica 2012, 97(4):516-23. 